\title{
16S rRNA-, GroEL- and MucZ-based assessment of the taxonomic position of 'Rickettsiella melolonthae' and its implications for the organization of the genus Rickettsiella
}

\author{
Andreas Leclerque and Regina G. Kleespies \\ Federal Biological Research Centre for Agriculture and Forestry (BBA), Institute for Biological \\ Control, Heinrichstr. 243, 64287 Darmstadt, Germany
}

Correspondence

Andreas Leclerque

leclerque@hotmail.com

\begin{abstract}
'Rickettsiella melolonthae' is an intracellularly multiplying bacterial pathogen of European cockchafers, Melolontha melolontha (Linnaeus, 1758) and Melolontha hippocastani (Fabricius, 1801) (Coleoptera: Scarabaeidae). We report the first determination of nucleotide sequences from this organism, i.e. the $16 \mathrm{~S}$ rRNA encoding rrs gene, the chaperonin encoding groEL gene and the mucZ gene encoding the orthologue of a capsule synthesis-inducing factor of Coxiella burnetii. Within the genus Rickettsiella, the pathotype 'Rickettsiella melolonthae' is currently classified as a synonym of the nomenclatural type species Rickettsiella popilliae. Previous sequencing of a $16 \mathrm{~S}$ rRNA gene from a different species, Rickettsiella grylli, has motivated the transfer of the entire genus from the alphaproteobacterial order Rickettsiales to the gammaproteobacterial order Legionellales, family Coxiellaceae. We investigated the validity of this taxonomic reorganization beyond the species Rickettsiella grylli by reconstructing the organismal phylogeny from comparisons of $16 \mathrm{~S}$ rRNA gene and GroEL and MucZ protein sequences from a selected set of alpha- and gammaproteobacteria as well as bacterial pathogens from the order Chlamydiales. Our analysis strongly supported the transfer of the genus Rickettsiella to the order Legionellales, but not its classification in one of the recognized families present in this order. Furthermore, our results substantiated inconsistencies in the internal organization of the genus. In particular, the currently accepted delineation of Rickettsiella species and the claimed synonymy of 'Rickettsiella melolonthae' with Rickettsiella popilliae are not simultaneously consistent with our findings.
\end{abstract}

The genus Rickettsiella (Philip, 1956) comprises intracellularly multiplying bacterial pathogens of insects, crustaceans and arachnids that were originally perceived as 'rickettsiae of insects' (Dutky \& Gooden, 1952; Krieg, 1955) and classified in the order Rickettsiales. An alternative assignment of these organisms to the order Chlamydiales has been judged less convincing (Weiss \& Moulder, 1984). Within the genus Rickettsiella, there are currently four species with validly published names: the type species Rickettsiella popilliae (Dutky \& Gooden, 1952), Rickettsiella grylli (Vago \& Martoja, 1963), Rickettsiella chironomi (Weiser, 1963) and Rickettsiella stethorae (Hall \& Badgley, 1957). However, according to Fournier \& Raoult (2005), there is not sufficient evidence available to warrant the consideration of Rickettsiella stethorae as a distinct species.

Abbreviations: ME, minimum evolution; ML, maximum-likelihood; MP, maximum-parsimony; NJ, neighbour-joining.

The GenBank/EMBL/DDBJ accession numbers for the rrs, groEL and mucZ gene sequences of 'Rickettsiella melolonthae' are EF408231, EF635858 and EF635859, respectively.
Numerous further pathotypes studied by electron microscopy have been suggested to represent synonyms of these species, while others still await conclusive species assignment. The pathotype 'Rickettsiella melolonthae' was discovered and first described as the causative agent of the 'Lorscher Erkrankung' (Lorsch disease) of white grubs of the European cockchafer species Melolontha melolontha (Linnaeus, 1758) and Melolontha hippocastani (Fabricius, 1801) (Coleoptera: Scarabaeidae) from near the town Lorsch, State of Hessen, Germany (Wille \& Martignoni, 1952). Originally named 'Rickettsia melolonthae' (Krieg, 1955), more detailed morphological and serological examinations (Krieg, 1958a, b, 1960; Huger \& Krieg, 1967) have led to its assignment as a synonym of the species Rickettsiella popilliae.

Strains of the genus Rickettsiella are generally considerably heterogeneous in terms of the cellular stages of the life cycle, the hosts and host tissues infected, the presence of protein crystals and the serological properties (for a review see Tanada \& Kaya, 1993 and Garrity et al., 2005). Detailed 
descriptions of developmental cycles point to a relatively close relationship between Rickettsiella popilliae and Rickettsiella grylli as compared with Rickettsiella chironomi (Götz, 1972) and reveal considerable similarities between the latter species and members of the genus Chlamydia (Federici, 1980). Nevertheless, a more recent study including Rickettsiella strains assigned to three of the species concluded from the results of DNA-DNA hybridization experiments, DNA G $+\mathrm{C}$ content ratios and DNA fine melting profiles that the genera Rickettsiella and Chlamydia are 'appropriately placed in different orders' (Frutos et al., 1994).

Sequencing of the 16S rRNA encoding rrs gene of a strain of Rickettsiella grylli revealed that it possessed the highest sequence similarity with the corresponding gene of Coxiella burnetii (Roux et al., 1997), i.e. a bacterial species that due to earlier 16S rRNA gene sequence analyses had been transferred from the alphaproteobacterial order Rickettsiales to the distantly related gamma subclass of the proteobacteria (Weisburg et al., 1989). As this finding calls into question the taxonomic classification of at least Rickettsiella grylli and reflects the original description of Rickettsiella popilliae as Coxiella popilliae, the entire genus Rickettsiella has recently been 'provisionally removed from the order Rickettsiales' and instead has been assigned to the gammaproteobacterial order Legionellales, family Coxiellaceae (Garrity et al., 2005). While this taxonomic reorganization is confirmed for Rickettsiella grylli by the recently published genome draft sequence (GenBank accession number NZ_AAQJ00000000) from one strain (strain TIGR) and partial 16S rRNA gene sequences (Cordaux et al., 2007) from three additional strains assigned to this species, the taxonomic positions of Rickettsiella popilliae and Rickettsiella chironomi remain uncertain due to the complete lack of sequence data from these species or from synonymous pathotypes such as 'Rickettsiella melolonthae'.

The determination of 16S rRNA gene sequences from three Rickettsiella pathotypes that, on the basis of electron microscopic studies, had not been assigned to any of the recognized species has produced conflicting results. On one hand, the rrs gene sequence from a bacterial endosymbiont of the tick Ixodes woodi (strain GSU) was found to display the highest similarity with that of Rickettsiella grylli (Kurtti et al., 2002). On the other hand, a presumed unnamed Rickettsiella strain infecting the terrestrial isopod Porcellio scaber that shows 'a high degree of morphological similarity with Rickettsiella grylli' (Drobne et al., 1999) and a putative rickettsiellal pathogen of the cockroach Blatta orientalis ('Rickettsiella blattae', Huger, 1963; 'Rickettsiella crassificans', Radek, 2000) were transferred to the newly created candidate genus 'Rhabdochlamydia' within the order Chlamydiales after the respective $16 \mathrm{~S}$ rRNA gene sequences had been determined (Kostanjsek et al., 2004; Corsaro et al., 2006). Irrespective of these conflicting results, monophyly has recently been claimed for the genus Rickettsiella (Cordaux et al., 2007).
The highly conserved Hsp60 chaperonin GroEL is distributed ubiquitously throughout the eubacterial lineage, appears functionally involved in bacterial endosymbiosis (Fares et al., 2004) and pathogenicity (Garduño et al., 1998) and has been used in molecular phylogenetic studies of proteobacterial endosymbionts of insects (Viale et al., 1994; Casiraghi et al., 2005). The mucZ gene product from C. burnetii induces capsule synthesis (mucoidy) when expressed in Escherichia coli (Zuber et al., 1995). MucZ proteins contain a C-terminal domain sharing significant similarity with the J domain of the bacterial Hsp40 cochaperone DnaJ, but are otherwise highly variable. Orthologues of the C. burnetii protein are identifiable to date only in a subset of the class Gammaproteobacteria including Legionella pneumophila. The $m u c Z$ gene has been used as a molecular marker to investigate the phylogeny of the genus Coxiella (Sekeyová et al., 1999). In a preliminary study using $C$. burnetii gene sequences as a query, we identified two identical rrs genes, as well as single copies of the $m u c Z$ and the groEL genes in the genome of Rickettsiella grylli. The finding of only a single copy of the groEL gene was in contrast to the situation usually found in chlamydial genomes where duplication of groEL genes is the rule (McNally \& Fares, 2007).

In this study, using the 16S rRNA gene and the GroEL and MucZ proteins as taxonomic markers, we report the first molecular sequence data for a Rickettsiella strain belonging to the type species Rickettsiella popilliae. We investigated whether these data: (i) corroborate the previously merely assumed assignment of Rickettsiella popilliae to the class Gammaproteobacteria, (ii) support the current family level classification of the genus Rickettsiella within the order Legionellales, and (iii) help to elucidate the internal taxonomic structure of the genus Rickettsiella, with particular emphasis on the validity of current species delineations and species-pathotype synonymies.

Genomic DNA of 'Rickettsiella melolonthae' strain BBA1806 was extracted from infected fat body tissue of diseased Melolontha hippocastani grubs collected in the Lorsch area, Germany, by using a standard protocol (Walsh et al., 1991) based on Chelex 100 resin (Bio-Rad). PCR amplification of an almost complete $16 \mathrm{~S}$ rRNA gene sequence was performed from the extract with primers fD1 and rP2 as described by Weisburg et al. (1991) using Phusion High-Fidelity DNA polymerase (Finnzymes) in a reaction running over 29 cycles of $15 \mathrm{~s}$ at $94{ }^{\circ} \mathrm{C}, 30 \mathrm{~s}$ at $50{ }^{\circ} \mathrm{C}$ and 2 min at $72{ }^{\circ} \mathrm{C}$. With identical PCR settings, the following pairs of degenerate oligonucleotides (MWG Biotech) were used to amplify internal fragments of the groEL (groELF1: 5'-GANGCNGGNGAYGGNACNACNAC; groELR1: 5'-ACYTTNATNACNGCNACNCCNCC) and the mucZ (mucZF1: 5'-GATGGACGTGTTTCTGAGGCAGARAT; mucZR1: 5'-CCTTTAGAGAGTAATTTATCNGGRTG) genes from the same template. For each gene, PCR products from three independent amplification reactions containing an appropriately sized product, as controlled by agarose gel electrophoresis, were purified by 
passage over a Qiaquick column (Qiagen) and sequenced on both strands. The six raw sequences were analysed, combined into a single consensus sequence and translated using the DNA Strider 1.3 program.

Similar nucleotide and amino acid sequence entries in the GenBank database were identified with the BLASTN and BLASTP software tools, respectively (Altschul et al., 1997). Sequence alignments were performed by means of the CLUSTAL $\mathrm{W}$ function (Thompson et al., 1994) of the MEGA 3.1 program (Kumar et al., 2004) using an International Union of Biochemistry (IUB) DNA and a Gonnet protein weight matrix. The TREE-PUZZLE 5.2 program (Schmidt et al., 2002) was used to estimate dataset-specific parameters. The most appropriate model of DNA sequence evolution was chosen according to the rationale outlined by Posada \& Crandall (1998), while amino acid sequence evolution models were directly chosen with the analysis tool available in TREEPUZZLE. Phylogenies were reconstructed with the maximumlikelihood (ML) method as implemented in the PHYML software tool (Guindon \& Gascuel, 2003), using the Hasegawa-Kishino-Yano (HKY) model of nucleotide substitution (Hasegawa et al., 1985) for the analysis of the $16 \mathrm{~S}$ rRNA gene and the JTT model (Jones et al., 1992) for the GroEL and MucZ amino acid sequence alignments. Additional minimum-evolution (ME) and neighbour-joining (NJ) 16S rRNA gene phylogenies were reconstructed in the MEGA 3.1 program under a Kimura two-parameter model of substitution (Kimura, 1980). In all cases, a $\Gamma$-distribution based model of rate heterogeneity (Yang, 1993) allowing for eight rate categories was assumed. A maximum-parsimony (MP) tree was constructed in MEGA 3.1 from the 16S rRNA gene sequence alignment on the basis of a max-mini branchand-bound algorithm. Tree topology confidence limits were explored in non-parametric bootstrap analyses over 1000 replicates. Trees were outgroup rooted by the respective sequence from the epsilonproteobacterium Myxococcus xanthus and arranged for more convenient comparison.

From each of the three sets of raw data generated from PCR products, reliable consensus sequences could be generated as the pairwise sequence similarities within a set were in no case $<99.5 \%$. Both protein coding consensus sequences were translated into partial sequences of 282 amino acids for the GroEL and 157 amino acids for the MucZ protein of 'Rickettsiella melolonthae'. When used as the query sequence of a BLASTP search for similar database entries, the respective orthologue from Rickettsiella grylli was identified as the best hit for both sequences. The $16 \mathrm{~S}$ rRNA consensus gene sequence derived from our data comprised 1462 nucleotides or approximately $95 \%$ of the rrs gene of 'Rickettsiella melolonthae' and, when used as BLASTN query, the best hits of $>95 \%$ sequence similarity at close to $100 \%$ query coverage were identified as the two available full-length 16S rRNA gene sequences from Rickettsiella grylli, together with the $16 \mathrm{~S}$ rRNA gene sequences from the above-mentioned Ixodes woodi endosymbiont (strain GSU) and a number of uncultured arthropod-associated bacteria.
The maximum-likelihood tree constructed from the alignment of bacterial rrs sequences (Fig. 1) displayed a clear distinction of two maximally supported branches comprising, respectively, members of the orders Chlamydiales, including the candidate genus 'Rhabdochlamydia', and Rickettsiales, including insect-associated Wolbachia, from a gammaproteobacterial branch. The latter, in turn, contained one major consistently structured sub-branch comprising, among others, the insect endosymbiotic genera Buchnera and Wigglesworthia in a sister position to the enterobacterial clade. These topological features were clearly supported in phylogenetic trees based on ME, MP and NJ analysis of $16 \mathrm{~S}$ rRNA gene sequences and the GroEL protein tree (data not shown). As mucZ genes are absent from most of these genera, the MucZ protein tree was non-informative at this level. The gammaproteobacterial branch was made up of several maximally supported clades representing single genera. Within this structure, the 16S rRNA gene sequence from 'Rickettsiella melolonthae' clustered firmly with the sequences from the two Rickettsiella grylli strains (Roux and TIGR) and the tick endosymbiont (strain GSU), thereby forming a presumed Rickettsiella clade located next to equally well supported Coxiella and Legionella clades. When included in the analysis, the three additional partial sequences from the isopod-pathogenic Rickettsiella grylli strains formed an additional branch within this clade (data not shown). With a similarly positioned Rickettsiella clade receiving near optimal bootstrap support in all of the further phylogenetic trees presented in Fig. 2, both of the considered alternative assignments of 'Rickettsiella melolonthae', i.e. as either a member of the candidate genus 'Rhabdochlamydia' of the order Chlamydiales or as according to its original classification in the order Rickettsiales, can be ruled out, while the co-assignment of 'Rickettsiella melolonthae' and Rickettsiella grylli is positively corroborated. This result is consistent with the monophyly hypothesis for the genus Rickettsiella. However, as long as sequence data are still lacking for the third, and in this respect, probably most critical species, Rickettsiella chironomi, such a claim must seem premature. Our view is that, under the assumption of the claimed synonymy of 'Rickettsiella melolonthae' with Rickettsiella popilliae, the transfer of the rickettsiellae to the gammaproteobacterial order Legionellales appears justified for two of the three Rickettsiella species.

There are, however, further features of the phylogenetic trees presented that are not in congruence with the current classification of the genus Rickettsiella. Firstly, the ML tree based on 16S rRNA gene sequences places the Rickettsiella clade in a sister position to the Coxiella clade, whereas the trees in Fig. 2 instead show a sister relationship of the Rickettsiella and Legionella clades. Clearly, this finding does not lend support to the current recognized classification of the genus Rickettsiella in the family Coxiellaceae and with neither of the two alternative topologies receiving a convincing bootstrap support, it might thus be more parsimonious to renounce a hierarchy building family assignment. 


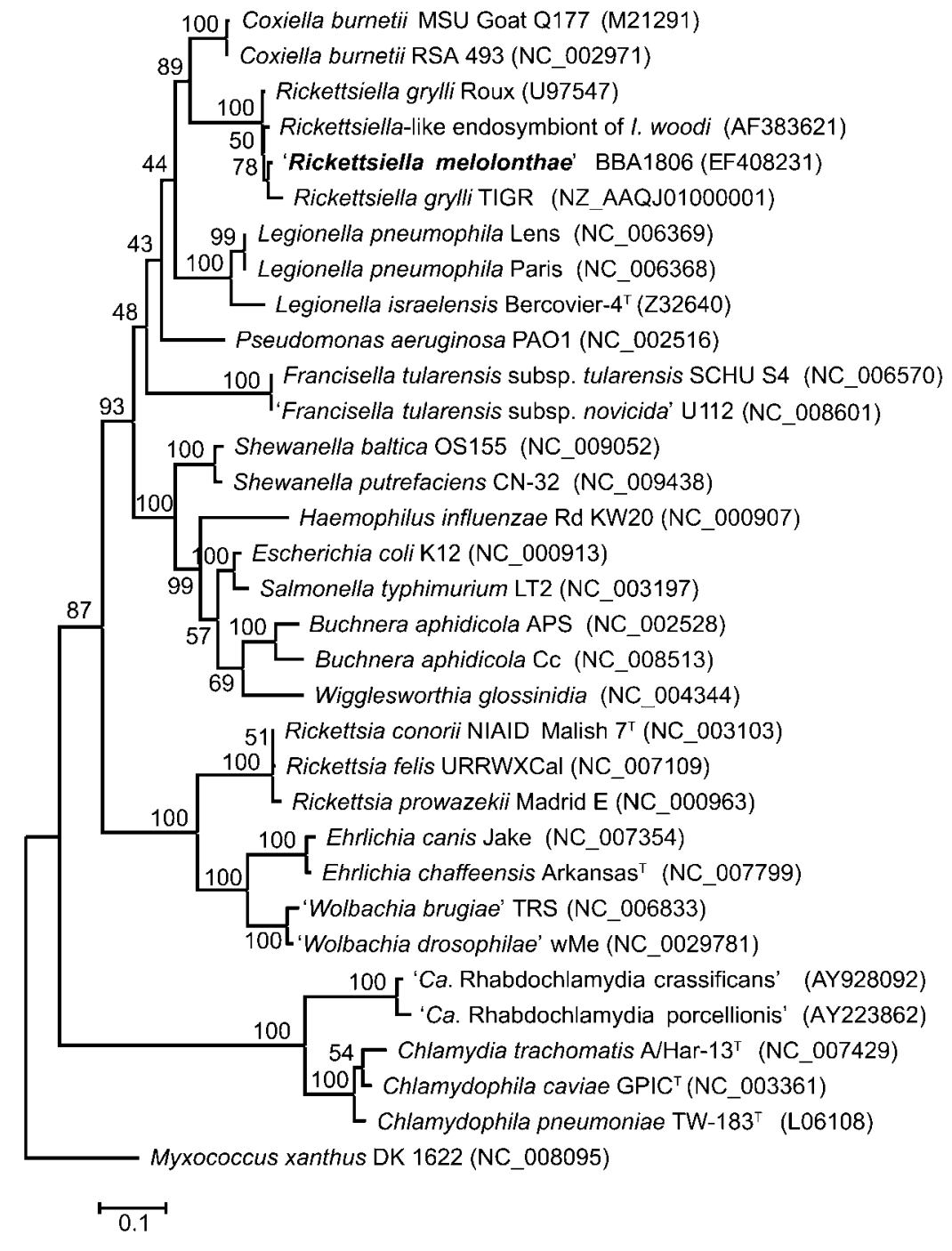

Fig. 1. Bacterial phylogeny reconstructed from 16S rRNA gene sequences by the ML method. Terminal branches are labelled by genus, species, and, where appropriate, the strain designation of the organism as well as the GenBank accession number of the respective sequence. Designations Rickettsiella grylli (Roux) and Rickettsiella grylli (TIGR) refer to the strain described by Roux et al. (1997) and to the genome project strain, respectively. Numbers on branches indicate bootstrap support values. Bar, 10\% relative sequence difference.
In addition, there is also incongruence with respect to the internal structure of the Rickettsiella clade as displayed in the $16 \mathrm{~S}$ rRNA gene trees presented. Irrespective of the method of phylogeny reconstruction applied, one of the two strains of Rickettsiella grylli is more closely related to 'Rickettsiella melolonthae' than to the second Rickettsiella grylli isolate, but under different reconstruction methods this is true for a different Rickettsiella grylli strain. The Rickettsiella clade thus lacks any topological structure reflecting the assumed species delineation. With pairwise sequence similarities of the respective $r r$ genes of 95-97\%, this is clearly not due to a lack of relevant phylogenetic information. On the contrary, the 16S rRNA gene sequences of the genus Rickettsiella appear rather heterogeneous if compared, for instance, with the $>99 \%$ sequence similarity found without exception for different isolates of C. burnetii (Stein et al., 1993) or to the $\geqslant 90 \%$, $\geqslant 95 \%$ and $\geqslant 98.5 \%$ sequence similarity thresholds employed, respectively, as criteria for the family, genus and species assignment of chlamydial strains (Everett et al., 1999). Application of these cut-off values would place each of the four Rickettsiella isolates (Rickettsiella grylli strains Roux and TIGR, 'Rickettsiella melolonthae' strain BBA1806 and Ixodes woodi endosymbiont strain GSU) in a different species, with the species concept thereby undercutting the pathotype concept, and, moreover, it would also remove the genus Rickettsiella from the family Coxiellaceae (data not shown). Due to their relatively short length, the further available partial 16S rRNA gene sequences from three additional strains of Rickettsiella grylli do not contribute any further conclusive data. The topological structure of the Rickettsiella clade resulting from our reconstructions of the 16S rRNA gene sequence phylogeny is, therefore, inconsistent with the currently accepted internal organization of this genus. The two studied strains of Rickettsiella grylli, Roux and TIGR, can be either placed in different species, with the consequence that the generally questionable pathotype-based system of species delineation has to be relinquished, or alternatively, if they are kept together in the same species, our results demand the inclusion of the strain BBA1806 of 'Rickettsiella melolonthae' (as well as the Ixodes woodi endosymbiont strain GSU) in this species. As 
(a)

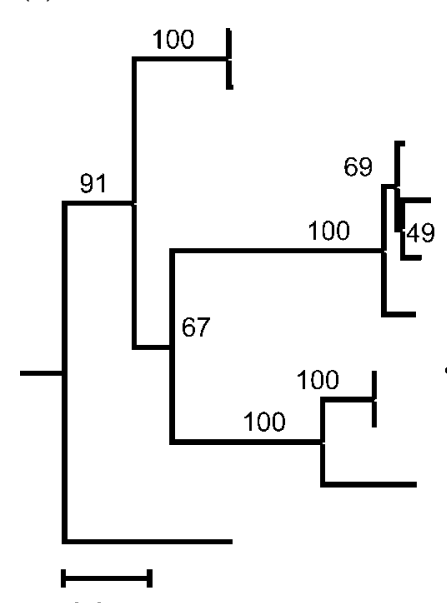

0.05 (b)

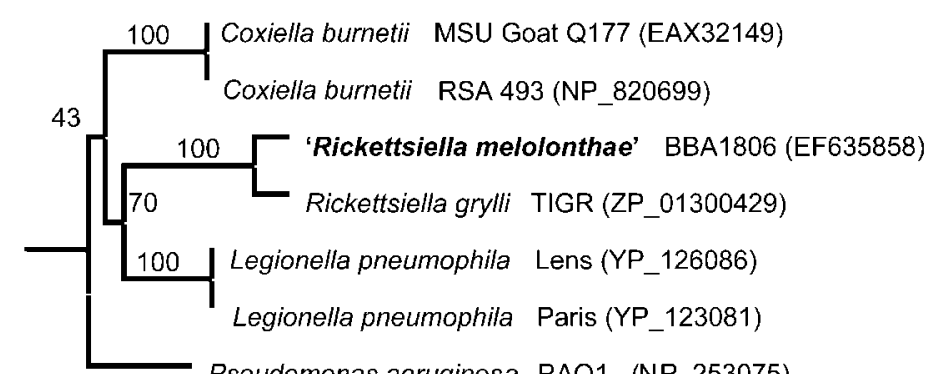

Pseudomonas aeruginosa PAO1 (NP 253075)

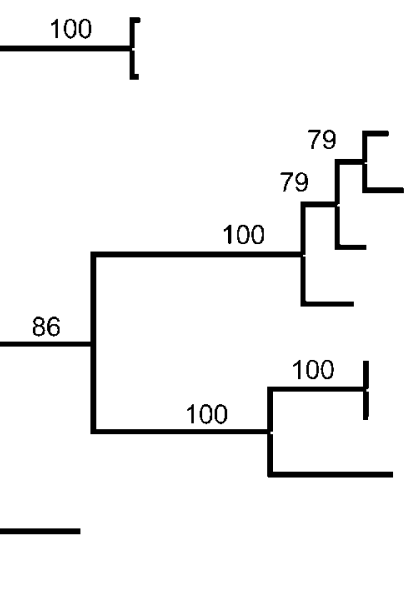

(c)

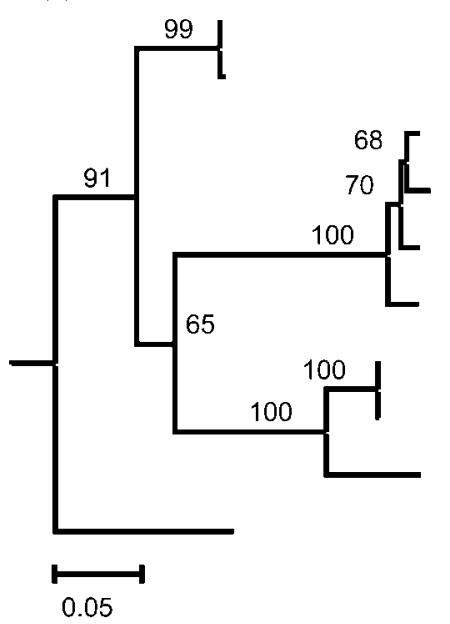

Coxiella burnetii MSU Goat Q177 (M21291)

Coxiella burnetii RSA 493 (NC_002971)

Rickettsiella grylli Roux (U97547)

70

Rickettsiella-like endosymbiont of I. woodi (AF383621)

'Rickettsiella melolonthae' BBA1806 (EF408231)

Rickettsiella grylli TIGR (NZ_AAQJ01000001)

Legionella pneumophila Lens (NC_006369)

Legionella pneumophila Paris (NC_006368)

Legionella israelensis Bercovier-4 ${ }^{\top}$ (Z32640)

Pseudomonas aeruginosa PAO1 (NC_002516)

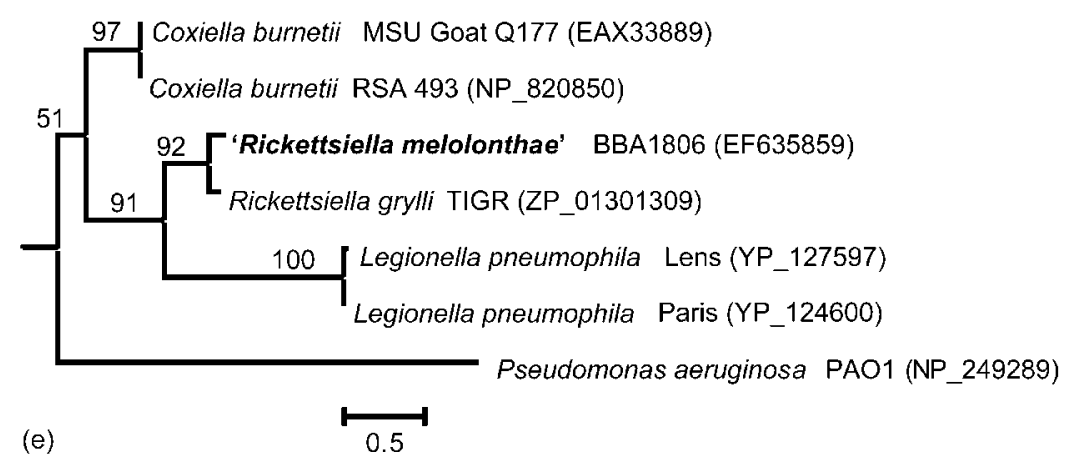

Fig. 2. Partial phylogenetic trees constructed from $16 \mathrm{~S}$ rRNA gene sequences by the ME (a), MP (b) and NJ (c) methods, as well as from GroEL (d) and MucZ (e) protein sequences by the ML method. The labels for the trees in a-c are given at the right hand side of the figures. Bars: $5 \%$ relative sequence difference ( $\mathrm{a}$ and $\mathrm{c}), 50$ nucleotide substitutions per site (b), $20 \%$ relative sequence difference (d) and $50 \%$ relative sequence difference (e). 
'Rickettsiella melolonthae' is a synonym for Rickettsiella popilliae, this means that the current delineation of the species Rickettsiella popilliae and Rickettsiella grylli would disappear. This last consequence could, in turn, be avoided by giving up the assumption of the synonymy of the species Rickettsiella popilliae and 'Rickettsiella melolonthae'.

In conclusion, our study lends firm support to the previous reorganization of the genus Rickettsiella as part of the gammaproteobacterial order Legionellales by furnishing the first molecular genetic evidence for the taxonomic position of a Rickettsiella pathotype classified in the species Rickettsiella popilliae. At the same time, our study emphasizes the weaknesses in the family level classification and internal organization of the genus. On one hand, the assignment of the genus Rickettsiella to the family Coxiellaceae is not supported by our findings and we feel that a hierarchically neutral assignment at the family level might be more appropriate. On the other hand, the currently accepted delineation of Rickettsiella species and the assumption of synonymy of 'Rickettsiella melolonthae' with Rickettsiella popilliae are not simultaneously consistent with our findings. Sequencing of the 16S rRNA gene from the Rickettsiella popilliae type strain will be the most straightforward way to properly decide which of these concepts will finally have to be abandoned. Meanwhile, the systematically most conservative, even if phylogenetically little convincing approach, consistent with our results would be to give up the morphologically and serologically comparatively well-founded claim of synonymy of Rickettsiella popilliae and 'Rickettsiella melolonthae'. Having secured the taxonomic position of the genus Rickettsiella, our study emphasizes the need for a reliable molecular taxonomic criterion for its internal organization.

\section{Acknowledgements}

We are highly indebted to Dr Kathrin Hartelt of the BadenWürttemberg State Health Office, District Government Stuttgart, Germany, for valuable advice concerning DNA extraction and her help with DNA sequencing as well as to Dr Gisbert Zimmermann of the Federal Biological Research Centre for Agriculture and Forestry (BBA) at Darmstadt, Germany, for critical reading of the manuscript.

\section{References}

Altschul, S. F., Madden, T. L., Schaffer, A. A., Zhang, J., Zhang, Z., Miller, W. \& Lipman, D. J. (1997). Gapped BLAST and PSI-BLAST: a new generation of protein database search programs. Nucleic Acids Res 25, 3389-3402.

Casiraghi, M., Bordenstein, S. R., Baldo, L., Lo, N., Beninati, T., Wernegreen, J. J., Werren, J. H. \& Bandi, C. (2005). Phylogeny of Wolbachia pipientis based on gltA, groEL and fts $Z$ gene sequences: clustering of arthropod and nematode symbionts in the F supergroup, and evidence for further diversity in the Wolbachia tree. Microbiology 151, 4015-4022.

Cordaux, R., Paces-Fessy, M., Raimond, M., Michel-Salzat, A., Zimmer, M. \& Bouchon, D. (2007). Molecular characterization and evolution of arthropod pathogenic Rickettsiella bacteria. Appl Environ Microbiol 73, 5045-5047.
Corsaro, D., Thomas, V., Goy, G., Venditti, D., Radek, R. \& Greub, G. (2006). 'Candidatus Rhabdochlamydia crassificans', an intracellular bacterial pathogen of the cockroach Blatta orientalis (Insecta: Blattodea). Syst Appl Microbiol 30, 221-228.

Drobne, D., Strus, J., Znidarsic, N. \& Zidar, P. (1999). Morphological description of bacterial infection of digestive glands in the terrestrial isopod Porcellio scaber (Isopoda, Crustacea). J Invertebr Pathol 73, 113-119.

Dutky, S. R. \& Gooden, E. L. (1952). Coxiella popilliae, n. sp., a rickettsia causing blue disease of Japanese beetle larvae. J Bacteriol 63, 743-750.

Everett, K. D. E., Bush, R. M. \& Andersen, A. A. (1999). Emended description of the order Chlamydiales, proposal of Parachlamydiaceae fam. nov. and Simkaniaceae fam. nov., each containing one monotypic genus, revised taxonomy of the family Chlamydiaceae, including a new genus and five new species, and standards for the identification of organisms. Int J Syst Bacteriol 49, 415-440.

Fares, M. A., Moya, A. \& Barrio, E. (2004). GroEL and the maintenance of bacterial endosymbiosis. Trends Genet 20, 413-416.

Federici, B. A. (1980). Reproduction and morphogenesis of Rickettsiella chironomi, an unusual intracellular procaryotic parasite of midge larvae. J Bacteriol 143, 995-1002.

Fournier, P.-E. \& Raoult, D. (2005). Genus II. Rickettsiella Philip 1956, $267^{\mathrm{AL}}$. In Bergey's Manual of Systematic Bacteriology, pp. 241-247. Edited by G. M. Garrity, D. J. Brenner, N. R. Krieg \& J. T. Staley. New York: Springer.

Frutos, R., Federici, B. A., Revet, B. \& Bergoin, M. (1994). Taxonomic studies of Rickettsiella, Rickettsia, and Chlamydia using genomic DNA. J Invertebr Pathol 63, 294-300.

Garduño, R. A., Garduño, E. \& Hoffman, P. S. (1998). Surfaceassociated Hsp60 chaperonin of Legionella pneumophila mediates invasion in a HeLa cell model. Infect Immun 66, 4602-4610.

Garrity, G. M., Bell, J. A. \& Lilburn, T. (2005). Family II. Coxiellaceae fam. nov. In Bergey's Manual of Systematic Bacteriology, pp. 237-247. Edited by G. M. Garrity, D. J. Brenner, N. R. Krieg \& J. T. Staley. New York: Springer.

Götz, P. (1972). 'Rickettsiella chironomi': An unusual bacterial pathogen which reproduces by multiple cell division. I Invertebr Pathol 20, 22-30.

Guindon, S. \& Gascuel, O. (2003). A simple, fast, and accurate algorithm to estimate large phylogenies by maximum likelihood. Syst Biol 52, 696-704.

Hasegawa, M., Kishino, H. \& Yano, T.-A. (1985). Dating of the human-ape splitting by a molecular clock of mitochondrial DNA. J Mol Evol 22, 160-174.

Hall, I. M. \& Badgley, M. E. (1957). A Rickettsial disease of larvae of species of Stethorus caused by Rickettsiella stethorae, n. sp. J Bacteriol 74, 452-455.

Huger, A. M. (1963). Eine Rickettsiose der Orientalischen Schabe, Blatta orientalis L., verursacht durch Rickettsiella blattae nov. spec. Naturwissenschaften 51, 22 (in German).

Huger, A. M. \& Krieg, A. (1967). New aspects of the mode of reproduction of Rickettsiella organisms in insects. J Invertebr Pathol 9, 442-445.

Jones, D. T., Taylor, W. R. \& Thornton, J. M. (1992). The rapid generation of mutation data matrices from protein sequences. Comput Appl Biosci 8, 275-282.

Kimura, M. (1980). A simple method for estimating evolutionary rates of base substitutions through comparative studies of nucleotide sequences. J Mol Evol 16, 111-120. 
Kostanjsek, R., Strus, J., Drobne, D. \& Avgustin, G. (2004). 'Candidatus Rhabdochlamydia porcellionis', an intracellular bacterium from the hepatopancreas of the terrestrial isopod Porcellio scaber (Crustacea: Isopoda). Int J Syst Evol Microbiol 54, 543-549.

Krieg, A. (1955). Licht- und elektronenmikroskopische Untersuchungen zur Pathologie der 'Lorscher Erkrankung' von Engerlingen und zur Zytologie der Rickettsia melolonthae nov. spec. Z Naturforsch $B$ 10b, 34-37 (in German).

Krieg, A. (1958a). Weitere Untersuchungen zur Pathologie der Rickettsiose von Melolontha spec. Z Naturforsch B 13b, 374-379 (in German).

Krieg, A. (1958b). Vergleichende taxonomische, morphologische und serologische Untersuchungen an insektenpathogenen Rickettsien. $Z$ Naturforsch B 13b, 555-557 (in German).

Krieg, A. (1960). Elektronenmikroskopische Untersuchungen zur Rickettsiose von Melolontha melolontha (L.) an Hand von UltraDünnschnitten. Z Naturforsch B 15b, 31-33 (in German).

Kumar, S., Tamura, K. \& Nei, M. (2004). MEGA3: integrated software for molecular evolutionary genetics analysis and sequence alignment. Brief Bioinform 5, 150-163.

Kurtti, T. J., Palmer, A. T. \& Oliver, J. H. (2002). Rickettsiella-like bacteria in Ixodes woodi (Acari: Ixodidae). J Med Entomol 39, $534-540$

McNally, D. \& Fares, M. A. (2007). In silico identification of functional divergence between the multiple groEL gene paralogs in Chlamydiae. BMC Evol Biol 7, 81.

Philip, C. B. (1956). Comments on the classification of the order Rickettsiales. Can J Microbiol 2, 261-270.

Posada, D. \& Crandall, K. A. (1998). Modeltest: testing the model of DNA substitution. Bioinformatics 14, 817-818.

Radek, R. (2000). Light and electron microscopic study of a Rickettsiella species from the cockroach Blatta orientalis. J Invertebr Pathol 76, 249-256.

Roux, V., Bergoin, M., Lamaze, N. \& Raoult, D. (1997). Reassessment of the taxonomic position of Rickettsiella grylli. Int J Syst Bacteriol 47, 1255-1257.

Schmidt, H. A., Strimmer, K., Vingron, M. \& von Haeseler, A. (2002). TREE-PUZZLE: maximum likelihood phylogenetic analysis using quartets and parallel computing. Bioinformatics 18, 502-504.

Sekeyová, Z., Roux, V. \& Raoult, D. (1999). Intraspecies diversity of Coxiella burnetii as revealed by com 1 and $m u c Z$ sequence comparison. FEMS Microbiol Lett 180, 61-67.
Stein, A., Saunders, N. A., Taylor, A. G. \& Raoult, D. (1993). Phylogenetic homogeneity of Coxiella burnetii strains as determined by $16 \mathrm{~S}$ ribosomal RNA sequencing. FEMS Microbiol Lett 113, 339-344.

Tanada, Y. \& Kaya, H. K. (1993). Insect Pathology, pp. 153-158. San Diego, CA: Academic Press.

Thompson, J. D., Higgins, D. G. \& Gibson, T. J. (1994). Clustal W: Improving the sensitivity of progressive multiple sequence alignment through sequence weighting, position-specific gap penalties and weight matrix choice. Nucleic Acids Res 22, 4673-4680.

Vago, C. \& Martoja, R. (1963). Une rickettsiose chez les Gryllidae (Orthoptera). C R Hebd Seances Acad Sci 256, 1045-1047 (in French).

Viale, A. M., Arakaki, A. K., Soncini, F. C. \& Ferreyra, R. G. (1994). Evolutionary relationships among eubacterial groups as inferred from GroEL (chaperonin) sequence comparisons. Int J Syst Bacteriol 44, 527-533.

Walsh, P. S., Metzger, D. A. \& Higuchi, R. (1991). Chelex 100 as a medium for simple extraction of DNA for PCR-based typing from forensic material. Biotechniques 10, 506-513.

Weisburg, W. G., Dobson, M. E., Samuel, J. E., Dasch, G. A., Mallavia, L. P., Baca, O., Mandelco, L., Sechrest, J. E., Weiss, E. \& Woese, C. R. (1989). Phylogenetic diversity of the rickettsiae. J Bacteriol 171, 4202-4206.

Weisburg, W. G., Barns, S. M., Pelletier, D. A. \& Lane, D. J. (1991). $16 \mathrm{~S}$ ribosomal DNA amplification for phylogenetic study. J Bacteriol 173, 697-703.

Weiser, J. (1963). Diseases of insects of medical importance in Europe. Bull World Health Organ 28, 121-127.

Weiss, E. \& Moulder, J. W. (1984). Order I. Rickettsiales Gieszczkiewicz 1939. In Bergey's Manual of Systematic Bacteriology, pp. 687-729. Edited by N. R. Krieg \& J. G. Holt. Baltimore, MD: Williams \& Wilkins.

Wille, H. \& Martignoni, M. E. (1952). Vorläufige Mitteilung über einen neuen Krankheitstypus beim Engerling von Melolontha vulgaris F. Schweiz Z Pathol Bakteriol 15, 470-473 (in German).

Yang, Z. (1993). Maximum-likelihood estimation of phylogeny from DNA sequences when substitution rates differ over sites. Mol Biol Evol 10, 1396-1401.

Zuber, M., Hoover, T. A. \& Court, D. L. (1995). Analysis of a Coxiella burnetii gene product that activates capsule synthesis in Escherichia coli: requirement for the heat shock chaperone DnaK and the twocomponent regulator RcsC. J Bacteriol 177, 4238-4244. 